\title{
IMPACTOS DA SÍNDROME DE BURNOUT NA QUALIDADE DE VIDA DOS PROFISSIONAIS DE ENFERMAGEM DA ATENÇÃO BÁSICA À SAÚDE
}

\section{IMPACTS OF BURNOUT SYNDROME ON THE QUALITY OF LIFE OF BASIC HEALTH CARE NURSING PROFESSIONALS}

\author{
Carlos Eduardo Barbosa Ramos ${ }^{1}$ \\ Jamilton Alves Farias ${ }^{2}$ \\ Maria Bernadete de Sousa Costa ${ }^{3}$ \\ Leila de Cássia Tavares da Fonseca ${ }^{4}$
}

\section{RESUMO}

Objetivo: Identificar o impacto da Síndrome de Burnout (SB) na qualidade de vida dos profissionais de enfermagem da Atenção Básica à Saúde. Métodos: Estudo exploratório e descritivo, com caráter quanti-qualitativo, desenvolvido com 52 profissionais da Rede de Atenção Básica à Saúde da Cidade de Bayeux, região metropolitana de João Pessoa-PB. Resultados: $13,5 \%$ das profissionais estudadas manifestaram características relacionadas à SB e $30,8 \%$ apresentaram predisposição para o desenvolvimento da mesma. Conclusão: O profissional acometido pela SB tende a apresentar diminuição em seu rendimento de trabalho e dificuldades na relação com a equipe de trabalho, refletindo o impacto negativo que a síndrome traz para a qualidade de vida.

DESCRITORES: Esgotamento Profissional. Atenção Primária à Saúde. Saúde do Trabalhador.

\begin{abstract}
Objective: To identify the impact of the Burnout Syndrome (SB) on the quality of life of nursing professionals in Basic Health Care. Methods: An exploratory and descriptive study, with quantitative and qualitative character, developed with 52 professionals from the Network of Basic Attention to Health of the city of Bayeux, metropolitan region of João Pessoa-PB. Results: $13.5 \%$ of the professionals studied showed characteristics related to $\mathrm{SB}$, and $30.8 \%$ presented a predisposition for its development. Conclusion: the professional affected by SB tends to present a decrease in his work income and difficulties in the relationship with the work team, reflecting the negative impact that the syndrome brings to the quality of life.
\end{abstract}

DESCRIPTORS: Occupational Exhaustion. Primary Health Care. Worker Health.

1- Enfermeiro. Residente do Programa de Residência Integrada Multiprofissional em Atenção a Saúde Hospitalar na Ênfase de Atenção a Saúde da Criança e do Adolescente da UFPB.

2- Enfermeiro. Doutor em Psicologia Clínica e da Saúde pela universidade de Salamanca - Espanha. Professor do Departamento de Enfermagem Clínica-DENC, da Universidade Federal da Paraíba-UFPB

3- Enfermeira. Doutora em Administração Sanitária e Hospitalar pela universidade de Extremadura-Espanha. Professora do Departamento de Enfermagem Clínica-DENC, da Universidade Federal da Paraíba-UFPB.

4- Enfermeira. Doutora em Enfermagem pela Universidade Federal da Paraíba. Professora do Departamento de Enfermagem Clínica-DENC, da Universidade Federal da Paraíba-UFPB. 
A s transformações que ocorrem no atual contexto da sociedade refletem em mudanças no processo de trabaIho e nos sujeitos envolvidos com ele. Desse modo, os profissionais necessitam desenvolver e aperfeiçoar suas habilidades, o que repercute tanto no domínio funcional, quanto no psicológico desses trabalhadores ${ }^{1}$.

Desta forma, a exposição a situações estressoras oriundas do ambiente de trabalho tem aumentado o surgimento de doenças de cunho emocional ${ }^{2}$. Nesse sentido, o estresse laboral pode estar relacionado tanto a fatores externos, ou seja, aqueles em que o profissional é atingido pelo contato com o meio, quanto com aqueles que são intrínsecos do trabalhador ${ }^{3}$.

Neste aspecto, estudos realizados sobre a Síndrome de Burnout (SB) entre os profissionais da Atenção Básica, ressaltam que, a saúde dos trabalhadores sofre influência de fatores psicossociais presentes no ambiente de trabalho ao qual estão inseridos. Dentre estes, destacam-se os fatores como a monotonia laboral, o relacionamento com os demais membros da equipe de profissionais e o sentimento de excesso de trabalho ${ }^{4-5}$.

Considerando que a Rede de Atenção Primária expõe o trabalhador ao contato diário com pessoas doentes e em sofrimento e que exige deste a execução de uma série de atribuições do serviço público para com a comunidade, esses profissionais que prestam assistência necessitam receber atenção especial quanto à manifestação da Síndrome de Burnout ${ }^{4}$.

$\mathrm{Na}$ área da saúde, a enfermagem é considerada uma das profissões de maior risco para o desenvolvimento de doenças emocionais e o enfermeiro é um profissional vulnerável ao surgimento da Síndrome de Burnout, uma vez que está envolvido num processo que lhe impõe uma fatigante rotina de trabalho ${ }^{5}$. Nesse contexto, observamos que a sobrecarga laboral, o baixo nível de suporte, os conflitos interpessoais, o contato com a morte e a preparação inadequada se constitui alguns dos fatores predisponentes para o desenvolvimento da síndrome ${ }^{2}$.

Na literatura, Burnout é uma palavra inglesa que traz como significado "queimar-se" ou "consumir-se". Comumente utilizada para referir-se a manifestações de sintomas como exaustão física e emocional e autodesvalorização presentes em profissionais da educação e da saúde, que no desempenho de suas atribuições estão em contato direto com pessoas ${ }^{6}$.

Uma das pesquisadoras pioneiras no assunto diz que a Síndrome de Burnout pode ser considerada um tipo de estresse do trabalho que surge em resposta a recorrente exposição social, principalmente quando envolve uma situação problema. Diferencia-se de outros tipos de estresse por ter origem na relação direta entre o trabalhador e as pessoas alvo do trabalho desenvolvido ${ }^{7}$. Com base nessa afirmativa, podemos dizer que essa síndrome não tem origem repentina, mas surge de forma gradual e desenvolve-se em fases que ao longo do tempo sofrem um progressivo aumento de intensidade ${ }^{2}$.

A Síndrome de Burnout é definida por um conjunto formado de três dimensões fortemente ligadas a atividade profissional: alto nível de exaustão emocional (EE); alto nível de despersonalização (DP) e baixo nível de realização profissional (RP) ${ }^{8}$. O modelo teórico 
de Maslach apresenta a dimensão EE como precursora do desenvolvimento as síndrome de Burnout, sendo seguida pelas dimensões DP e pela baixa RP9.

O diagnóstico e tratamento da Síndrome de Burnout podem ser dificultados por ser comumente confundida com outros tipos de estresse relacionados a problemas de origem pessoal $^{9}$. Muitas vezes, o diagnóstico pode ser dificultado pela falta de conhecimento do próprio trabalhador, por isso é importante que o profissional de enfermagem seja conhecedor desta síndrome ${ }^{5}$.

Considerada um problema social, a Síndrome de Burnout requer a busca de novos conhecimentos para o enfretamento de seus fatores desencadeantes, uma vez que, suas manifestações em profissionais de saúde podem comprometer a qualidade do atendimento oferecido ${ }^{8}$.

Estudos com os profissionais de enfermagem são importantes para que haja por parte dos órgãos ou instituições empregadoras, mecanismos de prevenção dos fatores desencadeantes do adoecimento emocional, visto que as repercussões negativas de tal adoecimento recaem não só sobre o próprio profissional, mas também sobre os pacientes, instituições e sociedade ${ }^{10}$.

$\mathrm{Na}$ literatura encontra-se autores tanto nacionais quanto internacionais que tem priorizado o estudo da Síndrome de Burnout em profissionais que atuam na rede de serviços de saúde, dessa forma, há necessidade de uma melhor avaliação das manifestações da referida síndrome nos diferentes campos de atuação desses profissionais ${ }^{11}$.

Justifica-se a realização deste estudo pelo diagnóstico dessa síndrome muitas vezes ser tardio e esta impactar significativamente na qualidade de vida dos profissionais acometidos, bem como na qualidade do serviço prestado por estes profissionais.

Considerando o exposto questiona-se: Qual o impacto a Síndrome de Burnout pode trazer para a qualidade de vida dos profissionais de enfermagem da Atenção Básica à Saúde? e quais os reflexos no atendimento aos usuários destes serviços? Para responder a estes questionamentos este estudo teve como objetivo identificar o impacto da Síndrome de Burnout na qualidade de vida dos profissionais de enfermagem da Atenção Básica à Saúde.

\section{MÉTODOS}

Trata-se de estudo do tipo exploratório e descritivo, com caráter quanti-qualitativo, desenvolvido com 52 profissionais nas unidades de saúde da Rede de Atenção Básica à Saúde da Cidade de Bayeux-PB, região metropolitana de João Pessoa-PB. Esta Rede dispõe de 28 Unidades de Saúde da Família (USF) distribuídas pelos bairros, das quais dez funcionam de forma integrada, dividindo a estrutura física do prédio, mas com equipe profissional própria de cada uma.

A população do estudo foi formada por 28 enfermeiros e 28 técnicos de enfermagem, perfazendo um total de 56 profissionais de enfermagem. Os critérios de inclusão para compor a amostra foram: estar atuando na Rede de Atenção Básica de Atenção á Saúde do Município no período da coleta de dados; concordar em participar do estudo e assinar o Termo de Consentimento Livre e Esclarecido.

O estudo foi realizado de acordo com a Resolução n. 466/2012, do Conselho Nacio- 
nal de Saúde que regulamenta as pesquisas que envolvem seres humanos no Brasil ${ }^{12}$. O projeto de pesquisa foi encaminhado e aprovado pelo Comitê de Ética e Pesquisa do Centro de Ciências da Saúde da Universidade Federal da Paraíba-UFPB, CAAE $n^{\circ}$ 55959816.9.0000.5188.

A coleta de dados foi realizada nos meses de junho e julho de 2016, no próprio local de trabalho dos profissionais de enfermagem, por meio de um instrumento com quatro blocos: O primeiro compreende um questionário sociodemográfico utilizado para conhecer o perfil dos profissionais participantes; o segundo constitui-se O Maslach Burnout Inventory - MBI avaliou as três dimensões da Síndrome de Burnout (exaustão emocional, despersonalização e redução da realização profissional). Esse instrumento foi desenvolvido pela pesquisadora Christina Maslach e é composto por 22 questões, onde nove referem-se à exaustão emocional, cinco são alusivas à despersonalização e oito são referentes à realização pessoal ${ }^{13}$. Para análise dos resultados foram utilizados os valores de referência do Núcleo de Estudos Avançados sobre Síndrome de Burnout - NEPASB (Quadro 1). ${ }^{14}$

O instrumento de Fatores Preditores e Sintomas da Síndrome de Burnout avaliou como os profissionais se comportaram quanto ao desenvolvimento dos sintomas avaliados. O WHOQOL-Bref avalia quatro domínios (Físico, Psicológico, Social e Meio Ambiente), foi utilizado para avaliar os resultados referentes à qualidade de vida dos sujeitos da pesquisa.

O banco de dados foi criado no software Microsoft Excel 2010, a partir daí, as variáveis foram analisadas com o auxílio do Statistical Package for Social Sciences SPSS versão 13.0. Foi utilizado ainda o teste estatístico não paramétrico de Qui-Quadrado, com nível de significância $p<0,005$. Após a análise estatística foram utilizados os resultados de maior significância para o estudo.

\section{RESULTADOS}

Com a análise das variáveis demográficas observamos que $100 \%$ das 52 profissionais estudadas são do sexo feminino. Em relação à idade destas profissionais, $42 \%$ (22) encontrava-se na faixa etária entre 31 a 40 anos de idade; $30,8 \%$ (16) possuíam de 18 a 30 anos e $26,9 \%$ (14) possuíam pelo menos 41 anos de idade. No que se refere ao estado civil, $46 \%$ (24) eram casadas, 32,7\% (17) eram solteiras e $21,2 \%$ (11) eram divorciadas. Quanto a maternidade, 58\% (30) afirmaram ter pelo menos um filho.

$\mathrm{Na}$ análise dos dados profissionais, foi evidenciado que $100 \%$ (52) das profissionais trabalham em turno integral e possuem jornada semanal de trabalho na Unidade de Saúde da Família de 40 horas.

Com relação ao tempo de trabalho, $51,9 \%$ (27) trabalhavam a menos de 5 anos e $40,4 \%$ (21) trabalhavam de 5 a 20 anos na Rede de Atenção Básica; 69,2\% (36) profissionais possuem renda mensal inferior a 5 salários mínimos. Foi identificado ainda que $83 \%$ (43) não possuem outro emprego e que $78,8 \%$ (41) afirmaram ter tirado férias no último ano.

O teste Qui-Quadrado não forneceu diferença estatisticamente significativa para: idade, estado civil, filhos, formação 
profissional e profissão no outro emprego. O teste apresentou significância para: renda mensal, tempo de trabalho, outro emprego e últimas férias gozadas.

No que tange a análise das dimensões da Síndrome de Burnout (Tabela 1) os valores encontrados demonstraram que $50 \%$ das profissionais possuíam um baixo nível de exaustão emocional, 51,9\% apresentaram baixo nível de despersonalização e 55,8\% tinham um alto nível de realização profissional.

De acordo com a escala do MBI apresentada no (Quadro 1) que apresentam as características que estabelecem diagnóstico para manifestação da Síndrome de Burnout, $15,4 \%$ das profissionais estudadas possuíam alta classificação para exaustão emocional, $13,5 \%$ alta classificação para despersonali-

TABELA 1. Classificação das dimensões da Síndrome de Burnout nas profissionais de enfermagem da ABS de Bayeux, 2016.

\begin{tabular}{l|cc|c}
\hline \multirow{2}{*}{ DIMENSÕES DA SB. } & \multicolumn{2}{|c|}{ Profissionais de Enfermagem } & \multirow{2}{*}{$\begin{array}{c}\text { Teste de } \chi^{2} \\
\text { Sig. p-valor }\end{array}$} \\
\cline { 2 - 3 } DIM-1: Exaustão emocional & $\mathrm{n}=52$ & Percentual (\%) \\
Baixo (0 - 15) & 26 & 50,009 \\
Médio (16 - 25) & 18 & 34,6 & \\
Alto (26 - 54) & 8 & 15,4 & \\
\hline DIM-2: Despersonalização & & & $\mathrm{p}=0,003$ \\
Baixo (0 - 2) & 27 & 51,9 & \\
Médio(3 - 8) & 18 & 34,6 & \\
Alto (9 - 30) & 7 & 13,5 & $\mathrm{p}=0,003$ \\
\hline DIM-3: Realização profissional & & & \\
Baixo (0 - 33) & 12 & 23,1 & \\
Médio (34 - 42) & 11 & 21,2 & \\
Alto (43 - 48) & 29 & 55,8 & \\
Total & 52 & 100,0 & \\
& & & \\
\hline
\end{tabular}

Fonte: Pesquisa direta, 2016.

QUADRO 1 - Valores da escala do MBI desenvolvidos pelo Núcleo de Estudos Avançados sobre a Síndrome de Burnout.

\begin{tabular}{|l|c|c|c|}
\hline \multirow{2}{*}{\multicolumn{1}{|c|}{ DIMENSÕES }} & \multicolumn{3}{|c|}{ PONTOS DE CORTE } \\
\cline { 2 - 4 } & BAIXO & MÉDIO & ALTO \\
\hline Exaustão emocional & $0-15$ & $16-25$ & $26-54$ \\
\hline Despersonalização & $0-02$ & $03-08$ & $09-30$ \\
\hline Realização profissional & $0-33$ & $34-42$ & $43-48$ \\
\hline
\end{tabular}

Fonte: Benevides-Pereira, 2002. 
zação e $23,1 \%$ possuíam baixa classificação para realização profissional.

Os resultados apresentados na Tabela 2, mostram que o teste de associação Qui-Quadrado aplicado para as três dimensões da Síndrome de Burnout segundo a categoria profissional não trouxe resultados estatísticos significativos para estas.

Constatou-se que 13,5\% (07) das profissionais apresentaram pelo menos duas dimensões alteradas, manifestando assim características relacionadas com a Síndrome de Burnout, sendo 4 enfermeiras e 3 técnicas de enfermagem. Verificou-se ainda que $30,8 \%$ (16) apresentaram predisposição para o desenvolvimento da Síndrome por estar com uma dimensão alterada, destas, 7 eram enfermeira e 9 eram técnicas de enfermagem.

Fazendo uma comparação entre as participantes por categoria profissional, constatamos que $15,4 \%$ das enfermeiras e $11,5 \%$ das técnicas de enfermagem manifestaram características relacionadas com a SB, enquanto que $26,9 \%$ das enfermeiras e $34,6 \%$ das técnicas de enfermagem apresentaram predisposição à síndrome.

Dentre os sintomas associados ao processo de trabalho (Tabela 3 ), os que mais se destacaram considerando sua presença pelo menos uma vez por semana foram: Cefaleia

TABELA 2. Classificação das Dimensões da Síndrome de Burnout segundo a Formação das Profissionais de Enfermagem da ABS de Bayeux,

\begin{tabular}{|c|c|c|c|c|c|c|c|}
\hline \multirow{3}{*}{ DIMENSÕES DA SB. } & \multirow{2}{*}{\multicolumn{2}{|c|}{ Total }} & \multicolumn{4}{|c|}{ Profissionais } & \multirow{3}{*}{$\begin{array}{c}\text { Teste de } \\
\quad \chi^{2} \\
\text { Sig. p-valor }\end{array}$} \\
\hline & & & \multicolumn{2}{|c|}{ Enfermeira } & \multicolumn{2}{|c|}{$\begin{array}{c}\text { Téc. } \\
\text { Enfermage } \\
\mathrm{m}\end{array}$} & \\
\hline & $n=52$ & $\%$ & $n$ & $\%$ & $n$ & $\%$ & \\
\hline Exaustão Emocional ${ }^{(b)}$ & & & & & & & $p=0,471$ \\
\hline Baixo $(0-15)$ & 26 & 50,0 & 11 & 42,3 & 15 & 57,7 & \\
\hline Médio (16 - 25) & 18 & 34,6 & 11 & 42,3 & 7 & 26,9 & \\
\hline Alto $(26-54)$ & 8 & 15,4 & 4 & 15,4 & 4 & 15,4 & \\
\hline Total & 52 & 100 & 26 & 100 & 26 & 100 & \\
\hline Despersonalização ${ }^{(b)}$ & & & & & & & $p=0,705$ \\
\hline Baixo $(0-2)$ & 27 & 51,9 & 12 & 46,2 & 15 & 57,7 & \\
\hline Médio (3 - 8) & 18 & 34,6 & 10 & 38,5 & 8 & 30,8 & \\
\hline Alto $(9-30)$ & 7 & 13,5 & 4 & 15,4 & 3 & 11,5 & \\
\hline Total & 52 & 100 & 26 & 100 & 26 & 100 & \\
\hline $\begin{array}{l}\text { Realização Profissional } \\
\text { (a) }\end{array}$ & & & & & & & $p=0,795$ \\
\hline Baixo $(0-33)$ & 12 & 23,1 & 5 & 19,2 & 7 & 26,9 & \\
\hline Médio (34 - 42) & 11 & 21,2 & 6 & 23,1 & 5 & 19,2 & \\
\hline Alto $(43-48)$ & 29 & 55,8 & 15 & 57,7 & 14 & 53,8 & \\
\hline Total & 52 & 100 & 26 & 100 & 26 & 100 & \\
\hline
\end{tabular}

Fonte: Pesquisa direta, 2016. 
43,1\% (22); Sentimento de cansaço mental $37,2 \%$ (19); Pouco tempo para si mesmo $33,3 \%$ (17); Dificuldades de sono 25,5\% (13); Dores nos ombros ou nuca 25\% (13); Perda ou excesso de apetite e Fadiga generalizada com $21,1 \%$ cada (11); Irritabilidade fácil 19,3\% (10); Dificuldade de memória e concentração 19,2\% (10); e Problemas alérgicos 15,4\% (8).

A Tabela 4 apresenta os resultados dos fatores preditores do burnout, onde verificou-se que quando considerando a sua presença em pelo menos uma vez por semana estes fatores comportaram-se da seguinte forma: $\mathrm{O}$ fator preditor 2 destacou-se estando presente em $86,5 \%$ (45), seguido pelos fator $5 \mathrm{com}$ $80,8 \%$ (42), fator 6 com $80,7 \%$ (42); fator 3 com 59.6\%(31); fator 4 com 48,1\%(25) e por fim o fator preditor 1 que representou $38,5 \%$ (20).

De acordo com os resultados do Gráfico 1, a avaliação feita pelas profissionais da Atenção Básica à Saúde em relação às

TABELA 3. Sintomas da Síndrome de Burnout pelas profissionais de enfermagem da ABS de Bayeux, 2016.

\begin{tabular}{|c|c|c|c|c|c|c|c|}
\hline \multirow{3}{*}{ SINTOMATOLOGIA } & \multicolumn{7}{|c|}{$\begin{array}{l}\text { ESCALA DE VALORES } \\
n=52\end{array}$} \\
\hline & 0 & 1 & 2 & 3 & 4 & 5 & 6 \\
\hline & $(\%)$ & $(\%)$ & $(\%)$ & $(\%)$ & $(\%)$ & $(\%)$ & $(\%)$ \\
\hline Cefaleia & 19,6 & 11,8 & 3,9 & 21,6 & 7,8 & 25,5 & 9,8 \\
\hline Irritabilidade fácil & 36,5 & 9,6 & 11,5 & 23,1 & 5,8 & 13,5 & 0,0 \\
\hline Perda ou excesso de apetite & 59,6 & 5,8 & 3,8 & 9,6 & 1,9 & 15,4 & 3,8 \\
\hline PA alta & 65,4 & 7,7 & 3,8 & 15,4 & 0,0 & 3,8 & 3,8 \\
\hline Dores nos ombros ou nuca & 34,6 & 9,6 & 13,5 & 17,3 & 0,0 & 21,2 & 3,8 \\
\hline Dor no peito & 80,8 & 7,7 & 1,9 & 5,8 & 0,0 & 0,0 & 3,8 \\
\hline Dificuldades de sono & 51,0 & 3,9 & 5,9 & 13,7 & 9,8 & 5,9 & 9,8 \\
\hline Sentimento de cansaço mental & 29,4 & 11,8 & 9,8 & 11,8 & 13,7 & 15,7 & 7,8 \\
\hline Dificuldades sexuais & 78,4 & 5,9 & 0,0 & 11,8 & 2,0 & 0,0 & 2,0 \\
\hline Pouco tempo para si mesmo & 17,6 & 5,9 & 21,6 & 21,6 & 7,8 & 11,8 & 13,7 \\
\hline Fadiga generalizada & 32,7 & 17,3 & 5,8 & 23,1 & 11,5 & 3,8 & 5,8 \\
\hline Pequenas infecções & 64,0 & 14,0 & 14,0 & 4,0 & 0,0 & 4,0 & 0,0 \\
\hline Aum. consumo de bebidas/cigarros & 92,3 & 0,0 & 5,8 & 0,0 & 1,9 & 0,0 & 0,0 \\
\hline Dific. de memória e concentração & 40,4 & 5,8 & 15,4 & 19,2 & 3,8 & 9,6 & 5,8 \\
\hline Problemas gastrointestinais & 53,8 & 9,6 & 13,5 & 11,5 & 3,8 & 3,8 & 3,8 \\
\hline Problemas alérgicos & 61,5 & 11,5 & 7,7 & 3,8 & 5,8 & 7,7 & 1,9 \\
\hline Estado de aceleração contínuo & 68,6 & 5,9 & 7,8 & 5,9 & 2 & 5,9 & 3,9 \\
\hline Sentir-se s/ vontade de começar nada & 61,5 & 1,9 & 13,5 & 7,7 & 3,8 & 3,8 & 7,7 \\
\hline Perda do senso de humor & 61,5 & 7,7 & 7,7 & 11,5 & 5,8 & 5,8 & 0,0 \\
\hline Gripes e resfriados & 32,7 & 34,6 & 17,3 & 7,7 & 1,9 & 3,8 & 1,9 \\
\hline Perda do desejo sexual & 78,8 & 1,9 & 9,6 & 5,8 & 0,0 & 1,9 & 1,9 \\
\hline
\end{tabular}

Fonte: Pesquisa direta, 2016. 
TABELA 4. Fatores Preditores da Síndrome de Burnout pelas profissionais de enfermagem da ABS de Bayeux, 2016.

\begin{tabular}{l|c|c|c|c|c|c|c}
\hline \multirow{2}{*}{$\begin{array}{l}\text { FATORES } \\
\text { PREDITORES }\end{array}$} & \multicolumn{7}{|c}{ ESCALA DE VALORES } \\
\cline { 2 - 8 } & 0 & 1 & 2 & 3 & 4 & 5 & 6 \\
\cline { 2 - 9 } & $(\%)$ & $(\%)$ & $(\%)$ & $(\%)$ & $(\%)$ & $(\%)$ & $(\%)$ \\
\hline FATOR1 & 38,5 & 5,8 & 9,6 & 7,7 & 5,8 & 25,0 & 7,7 \\
FATOR2 & 1,9 & 0,0 & 5,8 & 5,8 & 3,8 & 19,2 & 63,5 \\
FATOR3 & 13,5 & 21,2 & 1,9 & 3,8 & 0,0 & 23,1 & 36,5 \\
FATOR4 & 28,8 & 17,3 & 3,8 & 1,9 & 5,8 & 13,5 & 28,8 \\
FATOR5 & 1,9 & 1,9 & 5,8 & 9,6 & 1,9 & 21,2 & 57,7 \\
FATOR6 & 5,8 & 5,8 & 1,9 & 5,8 & 0,0 & 11,5 & 69,2 \\
\hline
\end{tabular}

Fonte: Pesquisa direta, 2016.

GRÁFICO 1 - Avaliação da qualidade de vida e satisfação com a saúde pelo instrumento WHOQOL-Bref para as profissionais de enfermagem da ABS de Bayeux, $2016(n=52)$.

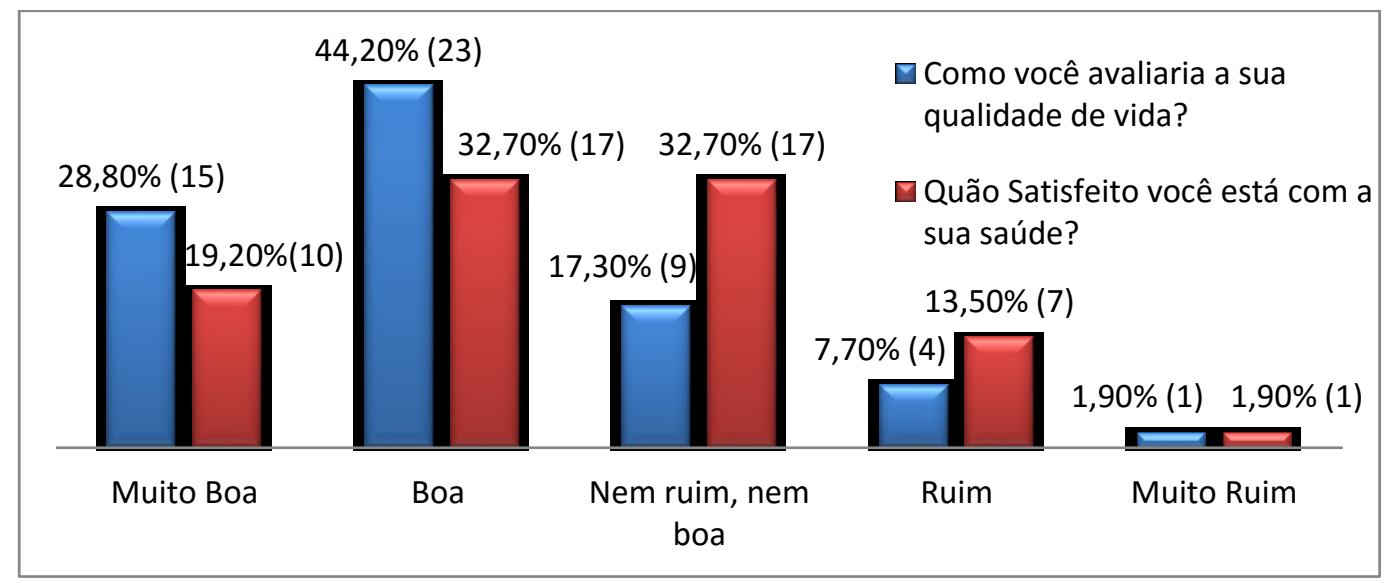

Fonte: Pesquisa direta, 2016.

questões: Como você avaliaria sua qualidade de vida? e Quão satisfeito (a) você está com a sua saúde?, demonstrou que $44,2 \%$ (23) dos profissionais avaliaram 'boa' sua qualidade de vida e $32,7 \%$ (17 das profissionais) avaliaram 'boa' sua saúde.

\section{DISCUSSÃO}

As respostas provenientes de uma avaliação onde o indivíduo responde a questionamentos sobre si, podem sofrer influências dos padrões sociais e ainda refletir autonegação, característica esta, presente na Síndrome de Burnout ${ }^{15}$.

A Síndrome de Burnout está relacionada ao trabalho e é fonte de danos pessoais e coletivos por comprometer não só a saúde do indivíduo, mas também interferir negativamente no cuidado oferecido por ele a sociedade ${ }^{4}$. 
Os transtornos de cunho psicossocial afetam a saúde física e mental dos indivíduos².

Vale ressaltar que muitos profissionais são pressionados pelas instituições e pelos próprios usuários dos serviços em busca de resultados, por outro lado, muitas vezes não recebem o devido apoio para alcançar os objetivos que lhes foram atribuídos ${ }^{16}$.

A desordem emocional surge a partir da relação desajustada entre os profissionais e seu ambiente de trabalho. As suas consequências podem manifestar-se de forma isolada e coletiva, o que requer um enfrentamento por parte da organização ${ }^{17}$.

A maioria dos enfermeiros estudados afirmaram estar satisfeitos com a sua profissão, isso demonstra que o real motivo de adoecimento não é a profissão em si, mas está relacionado com as fragilidades na organização do trabalho ${ }^{18}$, visto que, os aspectos que compõe a organização do trabalho de enfermagem são considerados os causadores de desgaste a estes profissionais ${ }^{2}$.

Os dados sociodemográficos e profissionais podem se constituir um fator de proteção ou de risco para o adoecimento. Os relacionamentos amorosos e o fato de possuir filhos ou não, pode influenciar significativamente a dimensão exaustão emocional ${ }^{19}$.

Este estudo evidenciou que $51,9 \%$ trabalhavam a menos de 5 anos. Este fato configura-se como um fator de risco para desordens emocionais relacionadas com o trabalho. De acordo com estudo realizado com um grupo de residentes um dos fatores considerados como predisponentes a SB foi o profissional estar em início de carreira ${ }^{6}$.

Durante o processo de formação, cada indivíduo constrói uma série de expectativas de realização pessoal e profissional. Muitas vezes ao adentrar no mercado de trabalho o profissional pode frustrar-se com as condições de trabalho oferecidas, esse fato pode explicar o risco de desenvolver a Síndrome de Burnout desde o início da carreira profissional.

O estresse emocional provocado por uma elevada jornada de trabalho pode resultar no desenvolvimento da SB que se dá ao longo do tempo ${ }^{4}$, a respeito desta afirmação foi observado que todas as profissionais entrevistadas dedicavam pelo menos 40 horas semanais de trabalho.

Como fator protetor relacionado ao perfil profissional, observamos que apenas 17\% (9) afirmavam possuir outro emprego o que eleva ainda mais o tempo semanal destinado ao trabalho e a maioria $78,8 \%$ tiveram período de férias no último ano.

Os resultados demonstraram que na sua maioria os profissionais da equipe de enfermagem participantes deste estudo, apresentaram baixo nível de exaustão emocional e despersonalização e alto nível de realização profissional. De forma divergente, o estudo realizado com os profissionais (enfermeiro, médico, odontólogo e assistente social) da Rede de Atenção Primária à Saúde de Aracaju-SE, mostrou que estes apresentaram em sua maioria exaustão emocional e despersonalização com nível de médio a alto e baixo nível de realização profissional ${ }^{4}$.

Este estudo demonstrou que 15,4\% das enfermeiras e $11,5 \%$ das técnicas de enfermagem são acometidas por características relacionadas com a Síndrome de Burnout. Por outro lado, em estudo realizado com profissionais de enfermagem das UBS e de um hospital, mostrou que a porcentagem 
de enfermeiros acometidos pela síndrome foi menor do que a de técnicos de enfermagem. Foi observado que mesmo cada um tendo um papel diferente dentro da equipe, estes apresentaram o mesmo risco para o adoecimento ${ }^{11}$.

Os profissionais de saúde necessitam conhecer a Síndrome de Burnout para que passem a identificar os fatores que colaboram para o adoecimento dentro de seu contexto de trabalho ${ }^{2-17}$. De modo semelhante, as instituições precisam contribuir com a prevenção e o tratamento de seus profissionais ${ }^{17}$.

Quanto aos fatores preditores da Síndrome de Burnout, para $69,9 \%$ das profissionais a afirmação (Tabela 4): na instituição onde atuo tenho a oportunidade de realizar um trabalho que considero importante está presente todos os dias. Nesse contexto, França e Ferrari ${ }^{20}$, dizem que o trabalho deve ser fonte de realização pessoal, onde o indivíduo amplie seus potenciais.

As relações conflituosas dentro da equipe de enfermagem e com os membros das equipes multiprofissionais de saúde contribuem para o surgimento do Burnout ${ }^{21}$. Por isso, é importante valorizar o trabalho em equipe dentro das instituições, bem como o papel de cada membro que a compõe. Para $57,7 \%$ a afirmação (Tabela 4): Percebo de forma evidente que existe respeito nas relações internas da instituição (na equipe de trabalho e entre coordenação de seus funcionários) está presente todos os dias no ambiente de trabalho.

Para a afirmação: Percebo que a instituição onde atuo é sensível aos funcionários, isto é, valoriza e reconhece o trabalho desenvolvido, assim como investe e incentiva o desenvolvimento profissional de seus funcionários, houve um equilíbrio entre as profissionais que escolheram a opção todos os dias e a opção nunca, $28,8 \%$ para cada. Os riscos ocupacionais podem estar relacionados com as pressões exercidas sobre o profissional e também com a instabilidade organizacional, bem como rigorosas regras institucionais ${ }^{2}$.

Em estudo realizado com enfermeiros da Atenção Básica à Saúde, quanto à identificação de sintomas relacionadas com a síndrome tema deste estudo o que mais se destacou foi irritabilidade fácil, onde 33,3\% dos profissionais afirmaram apresentar tal sintoma pelo menos uma vez por semana ${ }^{\text {. }}$ No presente estudo o sintoma irritabilidade fácil estava presente pelo menos uma vez por semana em $19,3 \%$, ocupando a $8^{\circ}$ posição entre os 10 que mais se destacaram.

A qualidade de vida possui forte ligação com a conquista dos anseios e as necessidades pessoais. Portanto, o ambiente de trabalho deve proporcionar sensação de bem-estar para os profissionais ${ }^{17}$.

Considerando um significado mais abrangente para a saúde, a qualidade de vida no trabalho deve ser entendida como sinônimo de saúde do trabalhador ${ }^{21}$. Quanto ao questionamento sobre o quão satisfeito as profissionais estavam com a sua saúde, houve um equilíbrio entre as respostas: "nem ruim, nem boa" e "boa", cada uma representada por $32,7 \%$.

Divergências encontradas em estudos sobre a qualidade de vida podem ser justificadas pelo fato da subjetividade dos dados, onde estes podem facilmente sofrer influências do tempo e do ambiente laboral dentro das instituições $^{22}$. Um desafio para os trabalhadores 
da enfermagem em seus diferentes campos de atuação profissional é a qualidade de vida que se tem no ambiente trabalho. Quando prejudicada, esta pode provocar o surgimento da Síndrome de Burnout ${ }^{21}$.

Alguns autores encontraram alto nível de satisfação dos profissionais com o trabalho, no entanto, estes pesquisadores demonstraram preocupação com a manutenção da qualidade de vida da população estudada. De forma semelhante, os sujeitos do presente estudo classificaram a sua qualidade de vida como boa. Portanto, compartilha-se da mesma preocupação em desenvolver estratégias para manutenção da qualidade de vida destes profissionais $^{22}$.

A Síndrome de Burnout, atualmente, vem sendo estudada em vários países uma vez que afeta relações socioculturais, administrativas e econômicas, indo mais além de que um problema psicológico ${ }^{21}$.

A ocorrência da SB pode ser alterada de acordo com a gestão de pessoas das instituições. Dessa forma, estratégias que permitam o crescimento e reconhecimento profissional podem prevenir os malefícios provocados pela referida síndrome ${ }^{23}$.

\section{CONCLUSÃO}

Profissionais da saúde, em especial os da equipe de enfermagem, são expostos diariamente a fatores estressores em seu ambiente de trabalho, independentemente de ser na assistência direta ao paciente ou até mesmo na área administrativa.

Os resultados apresentados revelaram que parte da população estudada manifesta os sinais e sintomas característicos da Síndrome de Burnout ou pelo menos está sob o risco de desenvolvê-la. O profissional acometido pela SB tende a apresentar diminuição em seu rendimento de trabalho e dificuldades na relação com a equipe de trabalho refletindo o impacto negativo que a síndrome traz para a qualidade de vida.

Embora a maioria das profissionais considere a sua qualidade de vida como boa, uma parcela relacionou uma série de sintomas à rotina de trabalho, isso confirma a influência negativa que os sintomas presentes no burnout apresentaram sobre a sua qualidade de vida.

É importante que os gestores tomem conhecimento que a prevenção de fatores precursores da SB trará benefícios não apenas para o profissional, mas também para a própria instituição na qual ele atua. Nesse sentido, uma das formas de apoio que poderiam ser realizadas por parte dos gestores seria oferecer melhores condições de trabalho e reconhecer às atividades desenvolvidas pelos profissionais.

Recomendamos o desenvolvimento de novos estudos que possam contribuir para o melhor conhecimento, em diferentes populações, e para a construção de estratégias eficazes, que atuem prevenindo, e até mesmo combatendo a síndrome que já esteja instalada. 


\section{REFERÊNCIAS}

1. França SPS, Martino MMFD, Aniceto EVS, Silva LL. Preditores da síndrome de burnout em enfermeiros de serviços de urgência pré-hospitalar. Acta Paul Enferm. 2012; 25(1):68-73.

2. Sanchez FFS, Oliveira R. Aspectos mediadores e desencadeadores da síndrome de burnout nos enfermeiros. Cuid Enferm. 2016;10(1): 61-7.

3. Meneghini F, Paz AA, Lautert L. Fatores ocupacionais associados aos componentes da síndrome de burnout em trabalhadores de enfermagem. Texto contexto enferm. 2011; 20(2):225-33.

4. Silva SCPS, Nunes MAP, Santana VR, Reis FP, Neto JM, Lima SO. A síndrome de urnout em profissionais da Rede de Atenção Primária à Saúde de Aracaju, Brasil. Ciênc saúde coletiva. 2015; 20(10):3011-20.

5. Cunha AP, Souza EM, Mello R. Os fatores intrínsecos ao ambiente de trabalho como contribuintes da síndrome de burnout em profissionais de enfermagem. Rev Pesquisa Cuid. Fundam. 2012; (Supl.):29-32.

6. Tavares KFA, Souza NVDO, Silva LD, Kestenberg CCF. Ocorrência da síndrome de burnout em enfermeiros residentes. Acta Paul Enferm. 2014; 27(3):260-265.

7. MASLACH, C.. Burnout. The cost of caring, Cambridge, M. A.: Malor. 2003.

8. Almeida LA, Medeiros IDS, Barros AG, Cristiane C, Martins F, Santos VEP. Fatores geradores da Síndrome de Burnout em profissionais da saúde. Rev Pesqui Cuid fundam. 2016; 8(3):4623-8.

9. Holmes ES, Santos SR, Farias JÁ, Costa MBS. Síndrome de burnout em enfermeiros na atenção básica: repercussão na qualidade de vida. Rev Pesqui Cuid Fundam. 2014; 6(4):1384-1395.

10. RISSARDO MP, GASPARINO RC. Exaustão emocional em enfermeiros de um hospital Público. Esc Anna Nery. 2013; 17(1):128-32.

11. Campos ICM, Angélico AP, Oliveira MS, Oliveira DCR. Fatores Sociodemográficos e Ocupacionais Associados à Síndrome de Burnout em Profissionais de Enfermagem. Psicol Reflex Crit. 2015; 28(4): 764-771.

12. Ministério da Saúde (BR), Conselho Nacional de Saúde. Resolução n. 466, de 12 de dezembro de 2012.
13. Tamayo MR, Troccoli BT. Construção e validação fatorial da Escala de Caracterização do Burnout (ECB). Estud Psicol.2009; 14(3):213-221.

14. Benevides-Pereira AM. Burnout: quando o trabalho ameaça o bem-estar do trabalhador. $1^{\mathrm{a}}$ ed. (Casa do Psicólogo). São Paulo, 2002.

15. Galdino MJQ,Martins JT, Haddad MCFL, Robazzi MLCC, Birolim MM. Síndrome de Burnout entre mestrandos e doutorandos em enfermagem. Acta Paul Enferm. 2016; 29(1):100-106.

16. Albuquerque FJB, Melo CF, Neto JLA. Avaliação da Síndrome de Burnout em Profissionais da Estratégia Saúde da Família da Capital Paraibana. Psicol Reflex Crit. 2012; 25 (3):542-49.

17. Kovaleski DF, Bressan A. A síndrome de Burnout em profissionais de saúde. Sau Transf Soc. 2012; 3(2):107-113.

18. Paula GS, Reis JF, Silvino ZR, Dutra VFD, Braga ALS, Cortez EA. O sofrimento psíquico do profissional de enfermagem. Rev Pesqui Cuid Fundam. 2012; (Supl.):33-36.

19. Ferreira NN, Lucca SR. Síndrome de burnout em técnicos de enfermagem de um hospital público do Estado de São Paulo. Rev Bras. Epidemiol. 2015; 18(1):68-79.

20. França FM, Ferrari R. Síndrome de Burnout e os aspectos sociodemográficos em profissionais de enfermagem. Acta Paul Enferm. 2012; 25(5): 743-748.

21. Lopes CCP, Ribeiro TP, Martinho NJ. Síndrome de Burnout e sua relação com a ausência de qualidade de vida no trabalho do enfermeiro. Enferm Foco. 2012; 3(2): 97-101.

22. Schmidt DRC, Paladini M, Biato C, Pais JD, Oliveira AR. Qualidade de vida no trabalho e burnout em trabalhadores de enfermagem de Unidade de Terapia Intensiva. Rev Bras Enferm. 2013; 66(1):13-17.

23. Sá MAS, Martins-Silva PO, Funchal B. Burnout: o impacto da satisfação no trabalho em profissionais de enfermagem. Psicol. Soc. 2014; 26(3): 664-674.

\section{CORRESPONDÊNCIA}

Carlos Eduardo Barbosa Ramos

Rua Santino Ambrósio Tonel, n.50 Bayeux,

Jardim São Severino. 58.306-300.

E-mail: carlos_eduardo92@hotmail.com. 